Canad. Math. Bull. Vol. 62 (2), 2019 pp. 223-230

http://dx.doi.org/10.4153/CMB-2018-018-0

(C) Canadian Mathematical Society 2018

\title{
Sharp Bertini Theorem for Plane Curves over Finite Fields
}

\author{
Shamil Asgarli
}

Abstract. We prove that if $C$ is a reflexive smooth plane curve of degree $d$ defined over a finite field $\mathbb{F}_{q}$ with $d \leq q+1$, then there is an $\mathbb{F}_{q}$-line $L$ that intersects $C$ transversely. We also prove the same result for non-reflexive curves of degree $p+1$ and $2 p+1$ when $q=p^{r}$.

\section{Introduction}

A classical theorem of Bertini states that if $X$ is a smooth quasi-projective variety in $\mathbb{P}^{n}$ defined over an infinite field $k$, then a general hyperplane section of $X$ is smooth. Specializing to the case when $C \subseteq \mathbb{P}^{2}$ is a smooth plane curve, it follows that there exists a line $L$ (defined over $k$ ) such that $L$ intersects $C$ transversely, meaning that $C \cap L$ consists of $d$ distinct geometric points where $d=\operatorname{deg}(C)$. But when $k=\mathbb{F}_{q}$ is a finite field, it is possible to have a smooth plane curve $C \subseteq \mathbb{P}^{2}$ such that every line $L$ defined over $\mathbb{F}_{q}$ is tangent to the curve $C$ (see Example 2.2). Moreover, Poonen's Bertini Theorem [8, Theorem 1.2] guarantees that such smooth curves, where all the $\mathbb{F}_{q}$-lines are tangent, do exist in every sufficiently large degree (see Example 2.3). With a view toward an effective version of Poonen's theorem, one can ask the following question.

Question 1.1 Suppose $C \subseteq \mathbb{P}^{2}$ is a smooth plane curve defined over $\mathbb{F}_{q}$. Let $d=$ $\operatorname{deg}(C)$. What conditions on $q$ and $d$ will ensure that there is a line $L \subseteq \mathbb{P}^{2}$ defined over $\mathbb{F}_{q}$ such that L meets $C$ transversely?

Let us call $L$ a good line if $L$ meets $C$ transversely. We expect that if $q$ is large with respect to $d$, then good lines will exist. Indeed, if $q \geq d(d-1)$, then the dual curve $C^{*}$ cannot be space-filling, i.e., $C^{*}\left(\mathbb{F}_{q}\right) \neq\left(\mathbb{P}^{2}\right)^{*}\left(\mathbb{F}_{q}\right)$. This is because $\operatorname{deg}\left(C^{*}\right) \leq$ $d(d-1) \leq q$ and a curve of degree of at most $q$ cannot go through all the points of $\left(\mathbb{P}^{2}\right)^{*}\left(\mathbb{F}_{q}\right)$. Any point in $\left(\mathbb{P}^{2}\right)^{*}\left(\mathbb{F}_{q}\right) \backslash C^{*}\left(\mathbb{F}_{q}\right)$ represents a good line $L \subseteq \mathbb{P}^{2}$ defined over $\mathbb{F}_{q}$. A generalization of this observation to higher dimensions is proved by Ballico [1, Theorem 1].

In this paper, we improve the quadratic bound $q \geq d(d-1)$ to the linear bound $q \geq d-1$.

Received by the editors April 17, 2018.

Published electronically May 18, 2018.

AMS subject classification: 14H50, 11G20, 14N05.

Keywords: Bertini theorem, transversality, finite field. 
Theorem 1.2 If $C$ is a smooth reflexive plane curve defined over $\mathbb{F}_{q}$ with $\operatorname{deg}(C) \leq$ $q+1$, then there is an $\mathbb{F}_{q}$-line $L$ such that $L$ intersects $C$ transversely.

The theorem is sharp in a sense that the statement cannot be improved to $q \geq$ $d-2$. There is a counter-example when $q=d-2$ (see Example 2.2). The "reflexive" assumption on $C$ is same as saying that $C$ has finitely many flex points (see Section 2). As a natural follow-up, we can ask the following question.

\section{Question 1.3 Does Theorem 1.2 hold when C is non-reflexive?}

In Section 3, we prove a partial result in this direction.

Theorem 1.4 Let $C$ be a smooth non-reflexive plane curve of degree $p+1$ or $2 p+1$ defined over $\mathbb{F}_{q}$ where $q=p^{r}$ with $r \geq 2$. Then there is an $\mathbb{F}_{q}$-line $L$ such that $L$ intersects C transversely.

Finally, in the last section of the paper (Section 4), we focus exclusively on Frobenius non-classical curves, which are non-reflexive curves of a special kind. As we will see, Question 1.3 in this case is equivalent to a statement about collinear $\mathbb{F}_{q}$-points on the curve.

Conventions In order to avoid various pathologies, we will assume throughout the paper that the characteristic of the field is $p>2$.

\section{Reflexive Curves}

In this section we review the theory of reflexive plane curves and prove Theorem 1.2. If $C$ is a plane curve defined over a field $k$, we can consider the Gauss map $\varphi: C \rightarrow\left(\mathbb{P}^{2}\right)^{*}$ that associates with each smooth point $p$ of $C$ its tangent line. The dual curve $C^{*}$ is defined to be the closure of $\varphi(C)$ inside $\left(\mathbb{P}^{2}\right)^{*}$. By looking at the Gauss map for the dual curve, we get $\varphi^{\prime}: C^{*} \rightarrow C^{* *}$. In what follows, we will identify $\mathbb{P}^{2}$ and $\left(\mathbb{P}^{2}\right)^{* *}$.

Definition 2.1 The curve $C$ is called reflexive if $C=C^{* *}$ and $\varphi^{\prime} \circ \varphi: C \rightarrow C^{* *}$ is the identity map.

A theorem of Wallace [9] asserts that $C$ is reflexive if and only if $\varphi$ is separable. As a result, all smooth plane curves in characteristic zero are reflexive. Recall that a point $P$ of $C$ is called a flex point if the tangent line at $P$ meets the curve $C$ at $P$ with multiplicity at least 3 . When $\operatorname{char}(k)=p>2$, we have the following characterization: $C$ is reflexive if and only if $C$ has finitely many flex points [7, Proposition 1.5].

Before we prove Theorem 1.2, here are some counter-examples of smooth curves $C$ where all the lines defined over $\mathbb{F}_{q}$ are tangent to $C$ (so that no good line exists).

Example 2.2 Let $C$ be a smooth plane curve with $\operatorname{deg}(C)=q+2$ such that $\# C\left(\mathbb{F}_{q}\right)=$ $\# \mathbb{P}^{2}\left(\mathbb{F}_{q}\right)$. Such curves exist, and have been extensively studied by Homma and Kim [6]. For such a curve $C$, every $\mathbb{F}_{q}$-line $L$ intersects $C$ at $q+2$ points (counted with multiplicity). But $q+1$ of these points are already accounted by the points of $L\left(\mathbb{F}_{q}\right)=$ 
$\mathbb{P}^{1}\left(\mathbb{F}_{q}\right)$. Thus, the residual intersection multiplicity results from $L$ being tangent to $C$ at one of the $\mathbb{F}_{q}$-points.

Example 2.3 Fix a finite field $\mathbb{F}_{q}$. Let $\left\{L_{1}, \ldots, L_{q^{2}+q+1}\right\}$ be all the $\mathbb{F}_{q}$-lines in the plane. Pick distinct (geometric) points $P_{i} \in L_{i}$ for each $i$. The condition that $C$ is tangent to $L_{i}$ at $P_{i}$ is a statement about vanishing of the first few coefficients in the Taylor expansion at these finitely many points. By applying Poonen's Bertini theorem with Taylor conditions [8, Theorem 1.2], there exists some $d_{0}$ such that for every $d \geq$ $d_{0}$, there exists a smooth plane curve $C \subseteq \mathbb{P}^{2}$ of degree $d$ such that $L_{i}$ is tangent to $C$ at $P_{i}$. In particular, all $\mathbb{F}_{q}$-lines $L \subseteq \mathbb{P}^{2}$ are tangent to $C$. A closer inspection of the proof reveals that the integer $d_{0}$ is in the order of $q^{2}$ (essentially because we imposed $q^{2}+q+1$ local conditions).

We will now prove the main theorem of this paper.

Theorem 1.2 If $C$ is a smooth reflexive plane curve defined over $\mathbb{F}_{q}$ with $\operatorname{deg}(C) \leq$ $q+1$, then there is an $\mathbb{F}_{q}$-line $L$ such that $L$ intersects $C$ transversely.

Proof Let $\Phi$ be the Frobenius map, defined on points by

$$
\Phi([X: Y: Z])=\left[X^{q}: Y^{q}: Z^{q}\right]
$$

We will write $T_{P}(C)$ for the tangent line to $C$ at a (geometric) point $P$. Set

$$
N=\#\left\{P \in C\left(\overline{\mathbb{F}_{q}}\right): \Phi(P) \in T_{P}(C)\right\},
$$

which is finite, because $C$ is reflexive [4]. Let $d=\operatorname{deg}(C)$. The following inequality is proved in [5, Theorem 8.41]:

$$
2 \cdot \# C\left(\mathbb{F}_{q}\right)+N \leq d(q+d-1)
$$

under the assumption that $C$ has finitely many flex points and that characteristic of the field is $p>2$. This is the step where we use the hypothesis that $C$ is reflexive.

Assume, to the contrary, that every $\mathbb{F}_{q}$-line is tangent to the curve $C$ at some (geometric) point. Let us divide these lines into two groups: if $L$ is tangent to $C$ at an $\mathbb{F}_{q}$-rational point, we will call $L$ a rational tangent. Otherwise, we will call $L$ a special tangent. Since every $\mathbb{F}_{q}$-line is tangent to $C$, and there are $q^{2}+q+1$ lines defined over $\mathbb{F}_{q}$, we get

$$
\#\{\text { rational tangents }\}+\#\{\text { special tangents }\}=q^{2}+q+1
$$

and

$$
\#\{\text { rational tangents }\} \leq \# C\left(\mathbb{F}_{q}\right)
$$

Now, if $L$ is a special tangent, it is tangent to the curve $C$ at a non- $\mathbb{F}_{q}$-point $P$. Then $L$ is also tangent to $C$ at $P, \Phi(P), \Phi^{2}(P), \ldots, \Phi^{e-1}(P)$ where $e=\left[k(P): \mathbb{F}_{q}\right]$ is the degree of the point $P$. Since $e \geq 2$, the line $L$ contributes at least 2 elements to $N$. As a result,

$$
2 \cdot \#\{\text { special tangents }\} \leq N \text {. }
$$


Combining all the inequalities above, we obtain that

$$
\begin{aligned}
q^{2}+q+1 & =\#\{\text { rational tangents }\}+\#\{\text { special tangents }\} \\
& \leq \# C\left(\mathbb{F}_{q}\right)+\frac{N}{2} \leq \frac{1}{2} d(q+d-1) \quad(\text { using }(*)) \\
& \leq \frac{1}{2}(q+1)(q+(q+1)-1)=\frac{1}{2}(q+1)(2 q)=q^{2}+q,
\end{aligned}
$$

which is a contradiction.

When $q=p$ is a prime, every smooth curve of degree at most $p$ is reflexive. Moreover, Pardini [7, Proposition] has shown that every smooth non-reflexive curve of degree $p+1$ (over any field of characteristic $p$ ) is projectively equivalent to the curve given by the equation $x y^{p}+y z^{p}+z x^{p}=0$. For this curve, many good lines exist. For instance, take two $\mathbb{F}_{p}$-points on the curve, and join them with a line $L$. Then $L$ will intersect $C$ transversely.

Consequently, we deduce the result for all smooth plane curves over $\mathbb{F}_{p}$ where $p$ is prime.

Corollary 2.4 If $C$ is a smooth plane curve defined over $\mathbb{F}_{p}$ with $\operatorname{deg}(C) \leq p+1$, where $p$ is a prime, then there is an $\mathbb{F}_{p}$-line $L$ such that $L$ intersects $C$ transversely.

\section{Non-reflexive Curves}

In this section, we will restrict attention to non-reflexive curves and prove Theorem 1.4.

Let $C \subseteq \mathbb{P}^{2}$ be a smooth non-reflexive curve defined over $\mathbb{F}_{q}$ with $q=p^{r}$ where $r \geq 2$. Pardini [7, Corollary 2.4] has shown that $C$ is defined by an equation of the form:

$$
a^{p} x+b^{p} y+c^{p} z=0
$$

where $a, b, c \in \mathbb{F}_{q}[x, y, z]$ are homogeneous polynomials of degree $t \geq 1$. In particular, $\operatorname{deg}(C)=t p+1$.

We establish a Bertini-type theorem for the case $t=1$ and $t=2$.

Theorem 1.4 Let $C$ be a smooth non-reflexive plane curve of degree $p+1$ or $2 p+1$ defined over $\mathbb{F}_{q}$ where $q=p^{r}$ with $r \geq 2$. Then there is an $\mathbb{F}_{q}$-line $L$ such that $L$ intersects C transversely.

Proof If $\operatorname{deg}(C)=p+1$, then $C$ is projectively equivalent to the curve given by the equation $x y^{p}+y z^{p}+z x^{p}=0$, for which many good lines $L$ exist (see the discussion before Corollary 2.4). For the rest of the proof, we will assume that $\operatorname{deg}(C)=2 p+1$. Since $C$ is non-reflexive, by [7, Corollary 4.3 ] the degree of the dual curve is

$$
\operatorname{deg}\left(C^{*}\right)=\frac{d(d-1)}{p}=\frac{(2 p+1)(2 p)}{p}=4 p+2 .
$$

For $p \geq 5$, we observe that $\operatorname{deg}\left(C^{*}\right)=4 p+2 \leq p^{2} \leq q$, so $C^{*}$ cannot contain all of $\left(\mathbb{P}^{2}\right)^{*}\left(\mathbb{F}_{q}\right)$, and hence any point $L \in\left(\mathbb{P}^{2}\right)^{*}\left(\mathbb{F}_{q}\right) \backslash C^{*}\left(\mathbb{F}_{q}\right)$ will be a desired line that intersects $C$ transversely. 
When $p=3$, the inequality $\operatorname{deg}\left(C^{*}\right)=4 p+2=14 \leq p^{r}=q$ still holds for $r \geq 3$. The only case that requires a separate analysis is $(p, r)=(3,2)$, which corresponds to degree $2 \cdot 3+1=7$ curve defined over $\mathbb{F}_{3^{2}}=\mathbb{F}_{9}$. The rest of the proof is devoted to studying this remaining case.

Let $C$ be a smooth non-reflexive curve of degree 7 defined over $\mathbb{F}_{9}$. Assume, to the contrary, that all the lines defined over $\mathbb{F}_{9}$ are tangent to $C$. Following the same terminology used in the proof of Theorem 1.2, we call $L$ a rational tangent if $L$ is tangent to $C$ at some $\mathbb{F}_{9}$-point. Otherwise, $L$ is called a special tangent. Since $C$ is non-reflexive, each tangent line $L$ must intersect the curve at the tangency point with multiplicity at least 3 ([7, Proposition 1.5]). Then the following hold.

(i) If $L$ is a rational tangent, then $L \cap C$ contains at most five $\mathbb{F}_{9}$-points.

(ii) If $L$ is a special tangent, then $L \cap C$ contains a conjugate pair of $\mathbb{F}_{81}$-points and a single $\mathbb{F}_{9}$-point. In symbols, $L \cap C=\left\{Q, Q^{\sigma}, P\right\}$, where $Q \in \mathbb{P}^{2}\left(\mathbb{F}_{81}\right) \backslash \mathbb{P}^{2}\left(\mathbb{F}_{9}\right)$ and $P \in \mathbb{P}^{2}\left(\mathbb{F}_{9}\right)$.

Consider the following incidence correspondence of points and lines:

$$
\mathcal{J}=\left\{(P, L): L \in\left(\mathbb{P}^{2}\right)^{*}\left(\mathbb{F}_{9}\right) \text { and } P \in(C \cap L)\left(\mathbb{F}_{9}\right)\right\} .
$$

Each $P \in C\left(\mathbb{F}_{9}\right)$ is contained in $q+1=10$ different $\mathbb{F}_{9}$-lines. Therefore, \#J $=$ $\# C\left(\mathbb{F}_{9}\right) \cdot 10$. On the other hand, using (i) and (ii) above, each special tangent $L$ contributes 1 point, while each rational tangent $L$ contributes at most 5 points to \#J. Thus, $\# \mathrm{~J} \leq S+5 R$ where $S$ and $R$ are the number of special and rational tangents, respectively. We deduce that $\# C\left(\mathbb{F}_{9}\right) \cdot 10 \leq S+5 R$. Since $\# C\left(\mathbb{F}_{9}\right) \geq R$, we get $10 R \leq S+5 R$, which implies $5 R \leq S$. Since $S+R=9^{2}+9+1=91$, we have $5(91-S) \leq S$, so that $S \geq \frac{5.91}{6}=75.8333 \ldots$. Thus, $S \geq 76$.

Next, take any rational tangent $L_{0}$. Every special tangent line intersects $L_{0}$ in one of its ten $\mathbb{F}_{9}$-points. Since $\frac{S}{10} \geq \frac{76}{10}>7$, there exists $P_{0} \in L_{0}\left(\mathbb{F}_{q}\right)$ such that there are at least 8 special tangent lines that pass through $P_{0}$. By looking at the ten $\mathbb{F}_{9}$-lines passing through $P_{0}$, we can estimate $\# C\left(\mathbb{F}_{9}\right)$ as follows. Each of the 8 special tangents will contribute at most 1 rational point, while the remaining (at most 2) rational tangents will contribute at most 5 rational points. Thus, one gets $\# C\left(\mathbb{F}_{9}\right) \leq 8+2 \cdot 5=18$. Consider the incidence correspondence

$$
\mathcal{J}=\left\{(P, L): L \text { is a special tangent and } P \in(C \cap L)\left(\mathbb{F}_{9}\right)\right\} .
$$

By (i) above, every special tangent contains exactly one $\mathbb{F}_{9}$-point of $C$, so that $\# \mathcal{I}=S$. As a result,

$$
S=\# \mathcal{J}=\sum_{P \in C\left(\mathbb{F}_{9}\right)} \#\{\text { special tangents passing through } P\} .
$$

Since

$$
\frac{S}{\# C\left(\mathbb{F}_{9}\right)} \geq \frac{76}{18}>4,
$$

there exists a point $P \in C\left(\mathbb{F}_{9}\right)$ such that at least 5 special tangents pass through $P$. Consider the corresponding line $P^{*}$ in the dual space $\left(\mathbb{P}^{2}\right)^{*}$, which consists of all lines passing through $P$. Let us look at the intersection of the line $P^{*}$ and the dual curve $C^{*}$ inside $\left(\mathbb{P}^{2}\right)^{*}$. The intersection has all the ten $\mathbb{F}_{9}$-points of $P^{*}$, since all the $\mathbb{F}_{9}$-lines are tangent to $C$. However, each of the special tangents is bitangent to $C$, so it 
is a node in $C^{*}$, and hence will contribute 2 to the intersection. It follows that $P^{*} \cap C^{*}$ has at least $5 \cdot 2+5=15$ intersections, contradicting the fact that $\operatorname{deg}\left(C^{*}\right)=14$.

Remark 3.1 As we saw above, the hardest part of the proof is the case $p=3$. This answers a question of Felipe Voloch, who asked, in a private communication, whether or not there exists a transverse line for a degree 7 smooth non-reflexive curve defined over $\mathbb{F}_{9}$. The small primes still persist when we try to extend Theorem 1.3 to nonreflexive curves of degree $3 p+1$. Indeed, if $C$ is a smooth non-reflexive curve of degree $3 p+1$, then

$$
\operatorname{deg}\left(C^{*}\right)=\frac{(3 p+1)(3 p)}{p}=9 p+3 \leq p^{2} \leq q
$$

for $p \geq 11$; the usual argument shows that $\left(C^{*}\right)\left(\mathbb{F}_{q}\right) \neq\left(\mathbb{P}^{2}\right)^{*}\left(\mathbb{F}_{q}\right)$, implying that good lines exist for $p \geq 11$. However, the main difficulty lies with the primes $p=3,5,7$.

\section{Connection to Frobenius Non-classical Curves}

In this section, we observe the implications of a Bertini-type theorem for a special class of non-reflexive curves, known as Frobenius non-classical curves.

Definition 4.1 Let $C \subseteq \mathbb{P}^{2}$ be a smooth plane curve defined over $\mathbb{F}_{q}$. Then $C$ is called Frobenius non-classical if $\Phi(P) \in T_{P}(C)$ for every $P$, where $T_{P}(C)$ is the tangent line to $C$ at the point $P$, and $\Phi: \mathbb{P}^{2} \rightarrow \mathbb{P}^{2}$ is the $q$-th power Frobenius map.

We should remark that the usual definition of Frobenius non-classical is stated differently (by looking at the order sequence of $C$ ), but the definition given above is equivalent in the case of smooth plane curves [4, Proposition 1].

Example Let $C$ be the curve defined over $\mathbb{F}_{q^{2}}$ by the equation

$$
x^{q+1}+y^{q+1}+z^{q+1}=0 .
$$

It can be checked that $C$ is a smooth Frobenius non-classical curve for $\mathbb{F}_{q^{2}}$.

If $C$ is a smooth Frobenius non-classical plane curve of degree $d$ defined over $\mathbb{F}_{q}$ where $q=p^{r}$, then it is known that $C$ is non-reflexive [4, Proposition 1] and $\sqrt{q}+1 \leq$ $d \leq \frac{q-1}{q^{\prime}-1}$, where $q^{\prime}$ is the generic order of contact of the curve with a tangent line [4, Propositions 5 and 6]. In particular, $\operatorname{deg}(C) \leq q-1$ always holds. So Question 1.3 is equivalent to the following.

Question 4.2 If $C$ is a smooth Frobenius non-classical plane curve defined over $\mathbb{F}_{q}$, does there exist an $\mathbb{F}_{q}$-line $L$ such that $L$ intersects $C$ transversely?

The existence of such a line $L$ can be verified for the curve $x^{q+1}+y^{q+1}+z^{q+1}=0$, and more generally, for the curve given by the equation

$$
x^{q^{n-1}+\cdots+q+1}+y^{q^{n-1}+\cdots+q+1}+z^{q^{n-1}+\cdots+q+1}=0,
$$


where $n \geq 2$. These curves are indeed smooth and Frobenius non-classical with respect to the field $\mathbb{F}_{q^{n}}[4$, Theorem 2].

If the Question 4.2 has an affirmative answer, then it implies that there is a line $L$ defined over $\mathbb{F}_{q}$ such that $L \cap C$ consists of $d=\operatorname{deg}(C)$ distinct $\mathbb{F}_{q}$-rational points. Indeed, if $L$ contains a non- $\mathbb{F}_{q}$-point $Q$, then we observe that $Q, \Phi(Q) \in T_{Q}(C)$ (since $C$ is Frobenius non-classical) and $Q, \Phi(Q) \in L$ (as $L$ is defined over $\mathbb{F}_{q}$ ), implying that $L=T_{Q}(C)$ is a tangent line. Thus, any good (transverse) line $L$ intersects $C$ at $\operatorname{deg}(C)$ distinct $\mathbb{F}_{q}$-points. This allows us to reformulate Question 4.2 as follows.

Question 4.3 If $C$ is a smooth Frobenius non-classical plane curve defined over $\mathbb{F}_{q}$, then does $C$ have $d=\operatorname{deg}(C)$ many $\mathbb{F}_{q}$-rational points on a line?

Question 4.3 is motivated by the fact that Frobenius non-classical curves have many $\mathbb{F}_{q}$-points. In fact, the $\mathbb{F}_{q}$-points on these curves have been used in $[2,3]$ to construct certain complete arcs in the plane. Moreover, the following theorem due to Hefez and Voloch [4, Theorem 1] gives the exact the number of $\mathbb{F}_{q}$-points on any smooth Frobenius non-classical plane curve.

Theorem 4.4 (Hefez-Voloch) If $C \subseteq \mathbb{P}^{2}$ is a smooth Frobenius non-classical curve of degree $d$ defined over $\mathbb{F}_{q}$, then $\# C\left(\mathbb{F}_{q}\right)=d(q-d+2)$.

We can apply Theorem 4.4 directly to get an estimate on the number of collinear points of $C$. Consider the incidence correspondence $\left\{(P, L): L \in\left(\mathbb{P}^{2}\right)^{*}\left(\mathbb{F}_{q}\right)\right.$ and $P \in$ $\left.(L \cap C)\left(\mathbb{F}_{q}\right)\right\}$. Since each $\mathbb{F}_{q}$-point $P$ is contained in $q+1$ lines,

$$
\# C\left(\mathbb{F}_{q}\right)(q+1)=\sum_{P \in C\left(\mathbb{F}_{q}\right)}(q+1)=\sum_{L} \#(L \cap C)\left(\mathbb{F}_{q}\right) .
$$

The sum on the right runs over all $q^{2}+q+1$ lines. Thus, an $\mathbb{F}_{q}$-line on average contains

$$
\frac{\# C\left(\mathbb{F}_{q}\right)(q+1)}{q^{2}+q+1}=\frac{d(q-d+2)(q+1)}{q^{2}+q+1}>\frac{d(q-d+2)}{q+1}>d\left(1-\frac{d}{q+1}\right)
$$

$\mathbb{F}_{q}$-points of $C$. As $q$ gets larger, this number approaches $d$. This heuristic suggests that Question 4.3 may have an affirmative answer.

Acknowledgements I would like to thank my advisor Brendan Hassett for the unwavering support and constant encouragement. I am especially grateful to Felipe Voloch for suggesting to investigate the case of degree 7 non-reflexive curves over $\mathbb{F}_{9}$, which led to Theorem 1.4. I also thank Dan Abramovich, Dori Bejleri, Herivelto Borges, Pol van Hoften, and Giovanni Inchiostro for insightful discussions and useful comments on the manuscript.

\section{References}

[1] E. Ballico, An effective Bertini theorem over finite fields. Adv. Geom. 3(2003), no. 4, 361-363. http://dx.doi.org/10.1515/advg.2003.020

[2] H. Borges, On complete (,$d$ )-arcs derived from plane curves. Finite Fields Appl. 15(2009), no. 1, 82-96. http://dx.doi.org/10.1016/j.ffa.2008.08.003

[3] M. Giulietti, F. Pambianco, F. Torres, and E. Ughi, On complete arcs arising from plane curves. Des. Codes Cryptogr. 25(2002), no. 3, 237-246. http://dx.doi.org/10.1023/A:1014979211916 
[4] A. Hefez and J. F. Voloch, Frobenius nonclassical curves. Arch. Math. 54(1990), no. 3, 263-273. http://dx.doi.org/10.1007/BF01188523

[5] J. W. P. Hirschfeld, G. Korchmáros, and F. Torres, Algebraic curves over a finite field. Princeton Series in Applied Mathematics, Princeton University Press, Princeton, NJ, 2008.

[6] M. Homma and S. J. Kim, Nonsingular plane filling curves of minimum degree over a finite field and their automorphism groups: supplements to a work of Tallini. Linear Algebra Appl. 438(2013), no. 3, 969-985. http://dx.doi.org/10.1016/j.laa.2012.08.032

[7] R. Pardini, Some remarks on plane curves over fields of finite characteristic. Compositio Math. 60(1986), no. 1, 3-17.

[8] B. Poonen, Bertini theorems over finite fields. Ann. of Math. 160(2004), no. 3, 1099-1127. http://dx.doi.org/10.4007/annals.2004.160.1099

[9] A. H. Wallace, Tangency and duality over arbitrary fields. Proc. London Math. Soc. 6(1956), 321-342. http://dx.doi.org/10.1112/plms/s3-6.3.321

Department of Mathematics, Brown University, Providence, RI 02912, USA

e-mail: shamil_asgarli@brown.edu 